\title{
Avant-propos et Épilogue du livre L'idéologie française
}

BHL

"Oui, je sais maintenant que la France, la France de ma culture, la France de ma mémoire, est aussi une France noire »

BHL

J'habite un pays étrange, extraordinairement mal connu, ceint d'une haute muraille de brumes, de fables et de mirages. J'y suis, nous y sommes tous, comme d'irréels rôdeurs, d'improbables vagabonds, déambulant à l'aveugle dans une mémoire ruinée, semée d'obscurités et des mystérieuses plages de silence. Je parle pourtant, on y parle même à tous vents, mais dans una langue opaque, langue de bois, langue de pierre, langue de bouches closes et d'oublieuses têtes, qu'on dirait occupées a tiser d'épais voiles de bruit et de sonores illusions. Cette langue voilée, c'est celle de notre Histoire. Et ce pays étrange, lointain, mal connu, dernier lieu d'exotisme, et tout cerné de brumes, c'est, en un mot, la France.

Car que savons-nous de la France ? Que nous a-t-on conté dans tous les hauts parages ou s'ourdissent ses glorieuses chansons de geste ? Que m'en a-t-on dit, à moi, tard venu dans le siècle, au lendemain des carnages qui manquèrent l'emporter ? On m'a dit à peu près que, de ces carnages, de ces orages inouïs, elle sortit innocente et pure de toute tache. On m'a patiemment enseigné que nous fûmes, nous Français, conçus immaculés, et miraculeusement immunisés contre les grands délires barbares qui ont ensanglanté l'époque. On nous a offert ainsi, dans un climat de liesse et de babils enchanteurs, une belle terre de cocagne qui n'aurait encensé le monde que de torrents de «bonheur», de «Liberté » de « Droits de l'Homme». Le fascisme ? Berlin. Le stalinisme? Moscou. La torture ? le racisme? Ailleurs, toujours ailleurs. Car ici, nous disait-on, nous sommes tous fils de Lumière, issus d'une
Histoire fabuleuse, peuple de communards, de dreyfusards, de maquisards, -nos hérauts avantageux dans l'ordre de l'honneur.

Or, il se trouve que, pour ma part, je suis las de vivre en rêve, schizophrène joyeux, imbécile satisfait, dans una France imaginaire ou je ne me reconnais pas. Nous sommes nombreux, je crois, à être fatigués de ces fables, de ces troubles amnésies, et de cette bonne conscience béate où se complaisent les faussaires. Trop de maquisards sont là, justement, entêtés à nous rappeler le visage d'une autre France qui, avant de les célébrer, a commencé par les proscrire et les mener au supplice. Trop de " dissidents ", rescapés des Goulags, arrivés d'un pas gourd en cette terre des "droits de l'homme » dont ils avaient ouï là-bas, au fond de leur hiver, la radieuse mythologie, et qui y trouvèrent bien souvent des portes closes, des regards embarrassés et d'infâmes crachats, sur leur face maculée encore des cendres de la veille. Le spectacle est trop insolent surtout, de ces autres hommes et femmes, "immigrés ", comme on dit, au séjour de «Liberté » et qui ne reçoivent généralement, en signe de bienvenue, qu'outrage, mépris, régulière humiliation, et une balle dans la peau, parfois, en guise de passeport. Oui, il est temps d'en finir. Il est urgent de faire taire la grinçante rengaine. Il est l'heure, enfin, de regarder la France en face.

C'est à cette urgence qu'entendent répondre les pages qui vont suivre. A cette généalogie de nos démons que j'ai voulu, ici, tenter de contribuer. Et cela, en posant, en ressassant, ces questions simples : n'y aurait-il pas, ici, au cœur même de la France, au fond de ce pays étrange, même si voilés de mutismes ou de légendes bavardes, un insistant secret, un obscur foyer 
de nuit, -dont nous serions les tributaires ? N'y aurait-il pas, au clos de nous-mêmes, a l'étouffée de nos mémoires, gravée aux tables de nos Lettres, quoique obstinément [sic.] celée à nos regards, une très ancienne plaie, -purulente jusqu'aujourd'hui, en nos terres et nos têtes ? Mieux : de quelles ombres, sur quels gouffres, sur quels buchers de mots et quels incendies d'Histoire, la douce France des profondeurs se bâtit-elle, -dont nous demeurons tous, bon gré mal gré, les fils et les acteurs ? Ces ombres, ces gouffres, je crains qu'ils n'aient des noms terribles, que l'on verra tout au long, et peu à peu, s'épeler. Et qui forceront à constater que la patrie des «droits de l'homme » est aussi la terre de deuil, l'inoubliable alambic, le ventre abominablement fécond où se sont enfantés quelques-uns des délires de l'Age où nous vivons.

Je ne dirais certes presque pas que j'ai pris plaisir à cette descente aux abîmes de l'idéologie française, J'ai eu peine, parfois, à réprimer une nausée face à ce quej'y découvrais et aux vapeurs qu'il m'y fallait respirer, Le voyage ne sera pas de tout repos, c'est sur aussi, pour les pétainistes impénitents, les nostalgiques de la honte, ou ceux qui, déjà, s'empressent à leur suite. Mais je crois, justement, qu'il n'est point d'autre chemin si nous voulons, décidément et pour de bon, interrompre la procession. Je suis convaincu qu'il n'est pas d'autre façon de conjurer le retour des fantômes que d'en apprendre l'inventaire et d'en identifier les hantises. L'Histoire, toute l'humble histoire des hommes est là, qui nous enseigne qu'un peuple amnésique, ignorant de ses oubliettes, est un peuple enchainé, voué à leurs relents. Et de fait, en cette heure où nous sommes et ou bourdonnent, de nouveau, tant de funestes présages, notre peuple en est bien là, qui ne semble toujours pas s'être vraiment résolu à arbitrer sans appel la querelle de ses deux traditions, -la France des résistants ou celle de la démission...

Autant dire que je ne risquerais pas à écrire, sije n'avais l'intention d'œuvrer ainsi, modestement, et depuis ma place, à ce nécessaire arbitrage. $\mathrm{Si}$ je n'avais l'espérance qu'un jour viendra, que je ne verrai peut-être pas, mais que d'autres verront, à qui je dédie ce livre, ou mon pays sera autre chose que ce suaire ou, présentement nous étouffons. Si je n'avais le sentiment, même incertain et mal fondé encore, que le ciel est plus haut déjà et que nous commençons de jouer le dernier acte de la nuit... Mais pour l'instant, et en attendant, la parole est à la mémoire, -et au « fascisme aux couleurs de la France » [...]

$* * *$

[...] Tel est donc le visage de cette idéologie française sur la piste de laquelle nous étions d'abord partis. Telle la constellation de signes qui la composent, que je ne prétends certes pas avoir tous élucidés, mais dont la langue, en tout cas, nous est maintenant plus familière. Et même si nombre d'ombres demeurent, en ces gîtes de textes dont je n'ai fait qu'arpenter quelques-uns des réseaux, au moins suis-je sûr qu'ils existent, que nous persistons d'y séjourner, -et qu'ils nous obligent plus rudement qu'on n'a coutume de le penser.

Oui, je sais maintenant que la France, la France de ma culture, la France de ma mémoire, est aussi une France noire. Je sais son visage d'ordure, la ménagerie de monstres qui y habitent, et ces paysages étranges où s'ouvrent parfois, en pleine lumière, des gouffres abominables. Je sais sa voix d'outre-brume, grinçante, meurtrière, et qui sonne si juste, pourtant, je l'ai assez dit, le plus incomparable de nos patois. Et ne sauraisje que cela, que j'aurais déjà beaucoup appris : et, par exemple, à ne plus ânonner le rituel et magique "fascisme ne passera pas », sans en observer les premiers pas aux lieux où il est déjà passé ; où il n’a pas même eu à passer puisqu'il $\mathrm{y}$ a toutes ses dynasties; où il n'aura pas même à revenir puisqu'il ne les a, en réalité, jamais désertés tout à fait.

J'ai appris également, et dans le même mouvement, à me défier de cette autre illusion, toujours diligente au défaut de la première, et qui, France pour France, ne consent à s'aviser du visage de cette France noire qu'en le fixant sous les traits d'une France imaginaire. France imaginaire, on l'a vu en effet, la France des " collabos " proprement dits, dont le rôle fut 
mince aux temps de la honte vichyssoise. France imaginaire, de même, le club moderne des nostalgiques de Drieu, de Doriot ou de Brasillach, hideux mais grotesques poupons de cire au musée Grévin de notre Histoire. Antifascistes imaginaires $\mathrm{du}$ coup, ces trop habiles régisseurs du théâtre de nos émois qui, régulièrement, une fois l'an, et dans un vacarme de grand guignol, viennent nous offrir en pâture des nazis purs et durs, criminels trop parfaits et prodiges de barbarie. Et péril réel alors, point $\mathrm{du}$ tout imaginaire, lui, en revanche, que ces oripeaux d'épouvante dont on drape la Bête française et ou se déguise, tout autant, son mufle très ordinaire : cette Bête, on ne le répétera jamais assez, n'est ni forcément ni évidemment la bête "immonde » de nos grand-peurs ; le propre discours a la traquer n'est pas celui de la pathologie, de la tératologie sociale, ou de l'exorcisme superstitieux ; mais c'est un discours plus fin, une écoute plus subtile, un regard de biais, -attentifs à des grondements discrets, à des sanctuaires mieux préservés et a d'incessants glissements, surtout, au fil de la modernité.

Car rien ne serait plus faux, je l'ai appris aussi, que de supposer ces voix tues, chues avec ceux qui les ont une fois portées, et indéfectiblement prisonnières des textes où elles sont d'abord apparues. On se souvient par exemple comment l'esprit du péguysme peut perdurer, avec ses fantasmes $\mathrm{d}^{\prime}$ " archies » et $\mathrm{d}^{\prime}$ " organicités », lors même que s'effacent, dans les consciences, ses signes de terre ou ses chiffres patriotes. On a vu comment les antiques matrices du socialisme primitif continuent de se décliner, en mille et une version, en autant de neuves devises, lors mémé que s'estompent les conditions de leur genèse. J'aurais pu insister davantage sur tel avatar de l'antisémitisme de gauche, si preste à jeter au loin ses guenilles usées et à endosser désormais, à peine travesti, le pimpant uniforme d'un « antisionisme » de bon aloi. Mais c'eût été redonder inutilement l'évidence. Et j'ai préféré mattarder aux vastes nappes de sens qui, midites ou non dites, gouvernent en sourdine tous ces énoncés disparates ou fluctuant au gré du temps; au filigrane muet qui les trame, les diffère, dicte leur dispersion et commande, en mémé temps, à leur régularités ; et si j’ai parlé des "dispositif ", c'est pour donner un nom à cette structure, a cette algèbre, qui, sous-tendant le désordre de leurs images, de leurs mutations spectaculaires, de leurs métamorphoses de surface, autorisent à reconnaitre la pérennité $\mathrm{d}$ 'une idéologie savante, riche en stratagèmes, -éternel phénix éternellement ressuscité de l'insistante saison des cendres.

De là encore que, si j'ai nettement souligné les formes les plus criminelles, les plus tumultueuses de cette idéologie, -l'antisémitisme justement ou, plus largement, le racisme-, je me suis gardé d'en faire l'unique objet ou même le pivot de mes analyses. Non pas, bien entendu, que je les croie accessoires. Pas davantage que je parie sur leur imminente extinction. Moins encore que je nie qu'elles soient toujours l'horizon, même reculé, du délire. Mais je pense simplement qu'elles ne sont, de ce délire, ni les seules figures, ni même les figures centrales. Et cela, simplement, parce que le fascisme français n'a pas de centre du tout. Qu'il a des sources assurément, mais pas d'unique foyer. Qu'il prend l'appui sur un ordre, mais n'a pas de dôme, de voute souveraine que le subjugue. Et que parler de "dispositif ", c'est viser un champ équivoque, une plaine aux mille voies, une langue aux infinies versions, dont il importait de dresser quelques-unes des plus diffuses tables, de nommer quelques-uns des plus ineffables quiproquos : les œuvres de la France noire, comme celles du Malin, procèdent toujours à bas bruit, à pas de loup, impromptues et inopinées ; et ce livre n'avait d'autre intention que d'exercer le regard a ses méridiens les plus troubles, les plus déroutants, et donc les plus redoutables.

Dira-t-on enfin que, procédant de la sorte, m'exerçant ainsi au guet et a tant de vigilance, je n'ai guère dégagé, au bout du compte, de " solutions positives »? Ou bien que, face à une menace si sournoise, à l'affût de tous parages, grande nef de Verbe louvoyant à l'estime de nos cultures, il nous reste, ici et maintenant, peu de redoutes, de recours pour résister ? C'est qui est exact, c'est que nos stratégies traditionnelles de résistance apparaissent bien chétives, et de bien peu de poids, face à une telle machinerie. C'est que l'idée même de « résistance » telle que nous 
avons coutume de la penser et que j'en ai moimême, ailleurs, élaboré le dessein, n'est plus tes pertinente, avec son parti pris de riposte et finalement de prudence, à l'ampleur de la menace. C'est qu'il est peut-être même l'heure d'aller au-delà de ces politiques «minimales », « ponctuelles », répondant au coup par le coup, au coup par le coup, á la guerre totale par la petite guerre d'usure, qui sont devenues toute notre doctrine, tous nos menus programmes, depuis que nous avons rompu les amarres aux grand systèmes d'autrefois. Et qu'il est temps, alors, d'imaginer autre chose, d'élaborer d'autres desseins, de réfléchir enfin aux lignes de cet antifascisme conséquent dont nous invoquons si souvent le patronage sans toujours prendre la peine de le penser jusqu'au bout.

Mais cet « antifascisme », justement, comment ne pas voir qu'il est là, lui aussi, tout près de nous, et également familier ? Qu'il s'impose tout autant, avec une égale évidence, ombre portée du délire, à l'aplomb de son ravage, lisible en creux de ses ubiquités ? Que de hautes figures en témoignent, hérauts vrais de nos Lettres, dont nous avons quelquefois eu à croiser la silhouette et qui, au long du siècle, en ont maintenu vaille que vaille l'ardente et difficile exigence ? La vérité, c'est qu'à une "idéologie » ne peut réellement s'opposer qu'une autre idéologie. C'est qu'à un lien social ne peut valablement répondre qu'un lien social adverse. C'est que si le fascisme est ce limbe d'une entière Cité, on ne peut l'ébranler, en conjurer l'avalanche, qu'en y opposant l'utopie d'une autre Cité, d'une autre houlette pour tenir ensemble les hommes. Ce lien social à inventer, cette Cité à retracer, cette houlette à retrouver, il faudra bien un jour se décider à leur rendre leur nom. A se soucier de leur lustre. A défouir leurs parvis, voilés d'obscurité. A revenir aux abords de leurs sources, surtout, presque exsangues au terme d'un siècle d'idéologie française. $D^{\prime}$ un mot : c'est la "démocratie », cette idée neuve, exotique, et étrangement cernée de brumes, dans la France des «Lumières », de la «Liberté », des « Droits de l'homme».

Bernard-Henri Lévy (1981). L'idéologie française. Paris : Editions Grasset \& Fasquelle, pp. 9-12 et 293-297. 